\title{
Probing the origin of cosmic-rays with extremely high energy neutrinos using the IceCube Observatory
}

M. G. Aartsen, ${ }^{2}$ R. Abbasi, ${ }^{29}$ M. Ackermann,,${ }^{45}$ J. Adams, ${ }^{15}$ J. A. Aguilar,${ }^{23}$ M. Ahlers,${ }^{29}$ D. Altmann, ${ }^{22}$

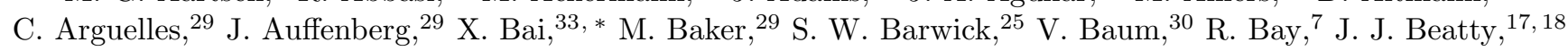
J. Becker Tjus, ${ }^{10}$ K.-H. Becker ${ }^{44}$ S. BenZvi, ${ }^{29}$ P. Berghaus,${ }^{45}$ D. Berley,${ }^{16}$ E. Bernardini,${ }^{45}$ A. Bernhard, ${ }^{32}$ D. Z. Besson, ${ }^{27}$ G. Binder ${ }^{8,7}$ D. Bindig, ${ }^{44}$ M. Bissok, ${ }^{1}$ E. Blaufuss,${ }^{16}$ J. Blumenthal,,${ }^{1}$ D. J. Boersma,${ }^{43}$ C. Bohm, ${ }^{36}$ D. Bose ${ }^{38}$ S. Böser, ${ }^{11}$ O. Botner,${ }^{43}$ L. Brayeur, ${ }^{13}$ H.-P. Bretz,${ }^{45}$ A. M. Brown, ${ }^{15}$ R. Bruijn, ${ }^{26}$ J. Casey ${ }^{5}$ M. Casier, ${ }^{13}$ D. Chirkin, ${ }^{29}$ A. Christov, ${ }^{23}$ B. Christy, ${ }^{16}$ K. Clark, ${ }^{39}$ F. Clevermann, ${ }^{20}$ S. Coenders, ${ }^{1}$ S. Cohen, ${ }^{26}$ D. F. Cowen,${ }^{42,41}$ A. H. Cruz Silva, ${ }^{45}$ M. Danninger, ${ }^{36}$ J. Daughhetee, ${ }^{5}$ J. C. Davis, ${ }^{17}$ M. Day, ${ }^{29}$ C. De Clercq,${ }^{13}$ S. De Ridder,${ }^{24}$ P. Desiati, ${ }^{29}$ K. D. de Vries,${ }^{13}$ M. de With,${ }^{9}$ T. DeYoung, ${ }^{42}$ J. C. Díaz-Vélez, ${ }^{29}$ M. Dunkman, ${ }^{42}$ R. Eagan, ${ }^{42}$ B. Eberhardt, ${ }^{30}$ J. Eisch, ${ }^{29}$ S. Euler, ${ }^{1}$ P. A. Evenson, ${ }^{33}$ O. Fadiran, ${ }^{29}$ A. R. Fazely, ${ }^{6}$ A. Fedynitch, ${ }^{10}$ J. Feintzeig, ${ }^{29}$ T. Feusels,${ }^{24}$ K. Filimonov,${ }^{7}$ C. Finley,${ }^{36}$ T. Fischer-Wasels,${ }^{44}$ S. Flis,${ }^{36}$ A. Franckowiak, ${ }^{11}$ K. Frantzen, ${ }^{20}$ T. Fuchs,${ }^{20}$ T. K. Gaisser, ${ }^{33}$ J. Gallagher, ${ }^{28}$ L. Gerhardt,${ }^{8,7}$ L. Gladstone,${ }^{29}$ T. Glüsenkamp, ${ }^{45}$ A. Goldschmidt ${ }^{8}$ G. Golup,${ }^{13}$ J. G. Gonzalez, ${ }^{33}$ J. A. Goodman, ${ }^{16}$ D. Góra,${ }^{22}$ D. T. Grandmont, ${ }^{21}$ D. Grant,${ }^{21}$

P. Gretskov, ${ }^{1}$ J. C. Groh, ${ }^{42}$ A. Groß,${ }^{32}$ C. Ha,${ }^{8,7}$ A. Haj Ismail,${ }^{24}$ P. Hallen, ${ }^{1}$ A. Hallgren,${ }^{43}$ F. Halzen, ${ }^{29}$ K. Hanson, ${ }^{12}$ D. Heereman, ${ }^{12}$ D. Heinen, ${ }^{1}$ K. Helbing, ${ }^{44}$ R. Hellauer, ${ }^{16}$ S. Hickford,${ }^{15}$ G. C. Hill, ${ }^{2}$ K. D. Hoffman, ${ }^{16}$ R. Hoffmann, ${ }^{44}$ A. Homeier, ${ }^{11}$ K. Hoshina, ${ }^{29}$ W. Huelsnitz,${ }^{16}$ P. O. Hulth,${ }^{36}$ K. Hultqvist,${ }^{36}$ S. Hussain, ${ }^{33}$

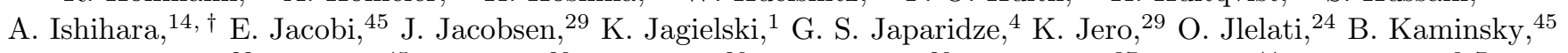
A. Kappes ${ }^{22}$ T. Karg, ${ }^{45}$ A. Karle,${ }^{29}$ M. Kauer, ${ }^{29}$ J. L. Kelley, ${ }^{29}$ J. Kiryluk, ${ }^{37}$ J. Kläs, ${ }^{44}$ S. R. Klein,,${ }^{8,7}$ J.-H. Köhne, ${ }^{20}$ G. Kohnen,${ }^{31}$ H. Kolanoski,${ }^{9}$ L. Köpke, ${ }^{30}$ C. Kopper, ${ }^{29}$ S. Kopper, ${ }^{44}$ D. J. Koskinen, ${ }^{19}$ M. Kowalski, ${ }^{11}$ M. Krasberg, ${ }^{29}$ A. Kriesten, ${ }^{1}$ K. Krings,${ }^{1}$ G. Kroll,${ }^{30}$ J. Kunnen, ${ }^{13}$ N. Kurahashi, ${ }^{29}$ T. Kuwabara, ${ }^{33}$ M. Labare ${ }^{24}$ H. Landsman, ${ }^{29}$ M. J. Larson, ${ }^{40}$ M. Lesiak-Bzdak, ${ }^{37}$ M. Leuermann, ${ }^{1}$ J. Leute, ${ }^{32}$ J. Lünemann, ${ }^{30}$ O. Macías, ${ }^{15}$ J. Madsen, ${ }^{35}$ G. Maggi, ${ }^{13}$ R. Maruyama, ${ }^{29}$ K. Mase,${ }^{14}$, 团 H. S. Matis, ${ }^{8}$ F. McNally,${ }^{29}$ K. Meagher, ${ }^{16}$ M. Merck, ${ }^{29}$ T. Meures, ${ }^{12}$ S. Miarecki, ${ }^{8,7}$ E. Middell, ${ }^{45}$ N. Milke, ${ }^{20}$ J. Miller, ${ }^{13}$ L. Mohrmann, ${ }^{45}$ T. Montaruli, ${ }^{23}$, 图 R. Morse ${ }^{29}$ R. Nahnhauer, ${ }^{45}$ U. Naumann, ${ }^{44}$ H. Niederhausen, ${ }^{37}$ S. C. Nowicki, ${ }^{21}$ D. R. Nygren, ${ }^{8}$ A. Obertacke,${ }^{44}$ S. Odrowski, ${ }^{21}$ A. Olivas, ${ }^{16}$ A. Omairat, ${ }^{44}$ A. O'Murchadha, ${ }^{12}$ L. Paul, ${ }^{1}$ J. A. Pepper,${ }^{40}$ C. Pérez de los Heros,${ }^{43}$ C. Pfendner, ${ }^{17}$ D. Pieloth,${ }^{20}$ E. Pinat,${ }^{12}$ J. Posselt, ${ }^{44}$ P. B. Price,${ }^{7}$ G. T. Przybylski ${ }^{8}$ L. Rädel,${ }^{1}$ M. Rameez ${ }^{23}$ K. Rawlins, ${ }^{3}$ P. Redl,${ }^{16}$ R. Reimann, ${ }^{1}$ E. Resconi, ${ }^{32}$ W. Rhode,${ }^{20}$ M. Ribordy,${ }^{26}$ M. Richman, ${ }^{16}$ B. Riedel,${ }^{29}$

J. P. Rodrigues, ${ }^{29}$ C. Rott,${ }^{38}$ T. Ruhe,${ }^{20}$ B. Ruzybayev ${ }^{33}$ D. Ryckbosch,${ }^{24}$ S. M. Saba, ${ }^{10}$ H.-G. Sander,${ }^{30}$

M. Santander, ${ }^{29}$ S. Sarkar, ${ }^{34}$ K. Schatto,${ }^{30}$ F. Scheriau, ${ }^{20}$ T. Schmidt, ${ }^{16}$ M. Schmitz, ${ }^{20}$ S. Schoenen, ${ }^{1}$

S. Schöneberg, ${ }^{10}$ A. Schönwald,${ }^{45}$ A. Schukraft,${ }^{1}$ L. Schulte, ${ }^{11}$ O. Schulz, ${ }^{32}$ D. Seckel,${ }^{33}$ Y. Sestayo,${ }^{32}$ S. Seunarine ${ }^{35}$ R. Shanidze ${ }^{45}$ C. Sheremata, ${ }^{21}$ M. W. E. Smith, ${ }^{42}$ D. Soldin,${ }^{44}$ G. M. Spiczak, ${ }^{35}$ C. Spiering ${ }^{45}$ M. Stamatikos, ${ }^{17}$, 田

T. Stanev, ${ }^{33}$ N. A. Stanisha, ${ }^{42}$ A. Stasik, ${ }^{11}$ T. Stezelberger, ${ }^{8}$ R. G. Stokstad,${ }^{8}$ A. Stößl, ${ }^{45}$ E. A. Strahler, ${ }^{13}$ R. Ström, ${ }^{43}$ G. W. Sullivan, ${ }^{16}$ H. Taavola ${ }^{43}$ I. Taboada, ${ }^{5}$ A. Tamburro ${ }^{33}$ A. Tepe ${ }^{44}$ S. Ter-Antonyan, ${ }^{6}$ G. Tešić ${ }^{42}$ S. Tilav ${ }^{33}$ P. A. Toale, ${ }^{40}$ M. N. Tobin,${ }^{29}$ S. Toscano,${ }^{29}$ E. Unger,${ }^{10}$ M. Usner,${ }^{11}$ S. Vallecorsa,${ }^{23}$ N. van Eijndhoven,,${ }^{13}$

A. Van Overloop, ${ }^{24}$ J. van Santen, ${ }^{29}$ M. Vehring, ${ }^{1}$ M. Voge, ${ }^{11}$ M. Vraeghe, ${ }^{24}$ C. Walck, ${ }^{36}$ T. Waldenmaier, ${ }^{9}$

M. Wallraff, ${ }^{1}$ Ch. Weaver, ${ }^{29}$ M. Wellons,${ }^{29}$ C. Wendt, ${ }^{29}$ S. Westerhoff,,${ }^{29}$ N. Whitehorn,,${ }^{29}$ K. Wiebe,${ }^{30}$

C. H. Wiebusch, ${ }^{1}$ D. R. Williams, ${ }^{40}$ H. Wissing,${ }^{16}$ M. Wolf,${ }^{36}$ T. R. Wood,${ }^{21}$ K. Woschnagg, ${ }^{7}$ D. L. Xu, ${ }^{40}$ X. W. Xu, ${ }^{6}$ J. P. Yanez, ${ }^{45}$ G. Yodh ${ }^{25}$ S. Yoshida, ${ }^{14, * *}$ P. Zarzhitsky, ${ }^{40}$ J. Ziemann, ${ }^{20}$ S. Zierke, ${ }^{1}$ and M. Zoll ${ }^{36}$

(IceCube Collaboration)

${ }^{1}$ III. Physikalisches Institut, RWTH Aachen University, D-52056 Aachen, Germany

${ }^{2}$ School of Chemistry 85 Physics, University of Adelaide, Adelaide SA, 5005 Australia

${ }^{3}$ Dept. of Physics and Astronomy, University of Alaska Anchorage, 3211 Providence Dr., Anchorage, AK 99508, USA

${ }^{4}$ CTSPS, Clark-Atlanta University, Atlanta, GA 30314, USA

${ }^{5}$ School of Physics and Center for Relativistic Astrophysics,

Georgia Institute of Technology, Atlanta, GA 30332, USA

${ }^{6}$ Dept. of Physics, Southern University, Baton Rouge, LA 70813, USA

${ }^{7}$ Dept. of Physics, University of California, Berkeley, CA 94720, USA

${ }^{8}$ Lawrence Berkeley National Laboratory, Berkeley, CA 94720, USA

${ }^{9}$ Institut für Physik, Humboldt-Universität zu Berlin, D-12489 Berlin, Germany

${ }^{10}$ Fakultät für Physik \&S Astronomie, Ruhr-Universität Bochum, D-44780 Bochum, Germany

${ }^{11}$ Physikalisches Institut, Universität Bonn, Nussallee 12, D-53115 Bonn, Germany

${ }^{12}$ Université Libre de Bruxelles, Science Faculty CP230, B-1050 Brussels, Belgium 
${ }^{13}$ Vrije Universiteit Brussel, Dienst ELEM, B-1050 Brussels, Belgium

${ }^{14}$ Dept. of Physics, Chiba University, Chiba 263-8522, Japan

${ }^{15}$ Dept. of Physics and Astronomy, University of Canterbury, Private Bag 4800, Christchurch, New Zealand

${ }^{16}$ Dept. of Physics, University of Maryland, College Park, MD 20742, USA

${ }^{17}$ Dept. of Physics and Center for Cosmology and Astro-Particle Physics,

Ohio State University, Columbus, OH 43210, USA

${ }^{18}$ Dept. of Astronomy, Ohio State University, Columbus, OH 43210, USA

${ }^{19}$ Niels Bohr Institute, University of Copenhagen, DK-2100 Copenhagen, Denmark

${ }^{20}$ Dept. of Physics, TU Dortmund University, D-44221 Dortmund, Germany

${ }^{21}$ Dept. of Physics, University of Alberta, Edmonton, Alberta, Canada T6G 2E1

${ }^{22}$ Erlangen Centre for Astroparticle Physics, Friedrich-Alexander-Universität Erlangen-Nürnberg, D-91058 Erlangen, Germany

${ }^{23}$ Département de physique nucléaire et corpusculaire,

Université de Genève, CH-1211 Genève, Switzerland

${ }^{24}$ Dept. of Physics and Astronomy, University of Gent, B-9000 Gent, Belgium

${ }^{25}$ Dept. of Physics and Astronomy, University of California, Irvine, CA 92697, USA

${ }^{26}$ Laboratory for High Energy Physics, École Polytechnique Fédérale, CH-1015 Lausanne, Switzerland

${ }^{27}$ Dept. of Physics and Astronomy, University of Kansas, Lawrence, KS 66045, USA

${ }^{28}$ Dept. of Astronomy, University of Wisconsin, Madison, WI 53706, USA

${ }^{29}$ Dept. of Physics and Wisconsin IceCube Particle Astrophysics Center,

University of Wisconsin, Madison, WI 53706, USA

${ }^{30}$ Institute of Physics, University of Mainz, Staudinger Weg 7, D-55099 Mainz, Germany

${ }^{31}$ Université de Mons, 7000 Mons, Belgium

${ }^{32}$ T.U. Munich, D-85748 Garching, Germany

${ }^{33}$ Bartol Research Institute and Department of Physics and Astronomy, University of Delaware, Newark, DE 19716, USA

${ }^{34}$ Dept. of Physics, University of Oxford, 1 Keble Road, Oxford OX1 3NP, UK

${ }^{35}$ Dept. of Physics, University of Wisconsin, River Falls, WI 54022, USA

${ }^{36}$ Oskar Klein Centre and Dept. of Physics, Stockholm University, SE-10691 Stockholm, Sweden

${ }^{37}$ Department of Physics and Astronomy, Stony Brook University, Stony Brook, NY 11794-3800, USA

${ }^{38}$ Department of Physics, Sungkyunkwan University, Suwon 440-746, Korea

${ }^{39}$ Department of Physics, University of Toronto, Toronto, Ontario, Canada, M5S $1 A 7$

${ }^{40}$ Dept. of Physics and Astronomy, University of Alabama, Tuscaloosa, AL 35487, USA

${ }^{41}$ Dept. of Astronomy and Astrophysics, Pennsylvania State University, University Park, PA 16802, USA

${ }^{42}$ Dept. of Physics, Pennsylvania State University, University Park, PA 16802, USA

${ }^{43}$ Dept. of Physics and Astronomy, Uppsala University, Box 516, S-75120 Uppsala, Sweden

${ }^{44}$ Dept. of Physics, University of Wuppertal, D-42119 Wuppertal, Germany

${ }^{45}$ DESY, D-15735 Zeuthen, Germany

(Dated: December 2, 2013)

We have searched for extremely high energy neutrinos using data taken with the IceCube detector between May 2010 and May 2012. Two neutrino induced particle shower events with energies around $1 \mathrm{PeV}$ were observed, as reported previously. In this work, we investigate whether these events could originate from cosmogenic neutrinos produced in the interactions of ultra-high energy cosmic-rays with ambient photons while propagating through intergalactic space. Exploiting IceCube's large exposure for extremely high energy neutrinos and the lack of observed events above $100 \mathrm{PeV}$, we can rule out the corresponding models at more than $90 \%$ confidence level. The model independent quasi-differential 90\% CL upper limit, which amounts to $E^{2} \phi_{\nu_{e}+\nu_{\mu}+\nu_{\tau}}=1.2 \times 10^{-7} \mathrm{GeV} \mathrm{cm}^{-2} \mathrm{~s}^{-1}$ $\mathrm{sr}^{-1}$ at $1 \mathrm{EeV}$, provides the most stringent constraint in the energy range from $10 \mathrm{PeV}$ to $10 \mathrm{EeV}$. Our observation disfavors strong cosmological evolution of the highest energy cosmic ray sources such as the Fanaroff-Riley type II class of radio galaxies.

PACS numbers: 98.70.Sa, 95.85.Ry, 95.55.Vj

* Physics Department, South Dakota School of Mines and Technology, Rapid City, SD 57701, USA

$\dagger$ Corresponding author.

aya@hepburn.s.chiba-u.ac.jp

$\ddagger$ Corresponding author. mase@hepburn.s.chiba-u.ac.jp

$\S$ also Sezione INFN, Dipartimento di Fisica, I-70126, Bari, Italy

ฯ NASA Goddard Space Flight Center, Greenbelt, MD 20771, USA

** Corresponding author. syoshida@hepburn.s.chiba-u.ac.jp 


\section{INTRODUCTION}

Cosmic neutrinos are expected to be produced in the interactions of high energy hadronic particles from cosmic accelerators with surrounding photons and matter. At $\mathrm{PeV}$ energies or greater, neutrinos are a unique tool for the direct survey of the ultra-high energy universe, because photons at these energies are highly attenuated by the cosmic microwave background (CMB). In addition to neutrinos directly produced in cosmic-ray sources, secondary neutrinos produced in the propagation of ultrahigh energy cosmic-rays (UHECRs) with energies reaching about $100 \mathrm{EeV}$ are expected. These "cosmogenic" neutrinos are produced by the Greisen-Zatsepin-Kuzmin (GZK) mechanism via interactions of UHECRs with the CMB and extragalactic background light (infra-red, optical, and ultra violet) [1 3]. A measurement of cosmogenic (or GZK) neutrinos probes the origin of the UHECRs because the spectral shapes and flux levels are sensitive to the redshift dependence of UHECR source distributions and cosmic-ray primary compositions [4, 5]. Neutrinos are ideal particles to investigate the origin of UHECRs since neutrinos propagate to the Earth essentially without deflection and absorption. The main energy range of the cosmogenic neutrinos is predicted to be around $100 \mathrm{PeV}-10 \mathrm{EeV}[\underline{6}$, 7]. In this extremely high energy (EHE) region, cosmogenic production is considered the main source of cosmic neutrinos.

A measurement of these EHE neutrinos requires a detection volume on the order of at least $1 \mathrm{~km}^{3}$ as their fluxes are expected to be very low, yielding approximately one event per year in such a volume [8, 9]. The IceCube Neutrino Observatory [10] at the geographical South Pole is the first cubic-kilometer scale neutrino detector. Its large instrumented volume as well as its omni-directional neutrino detection capability have increased the sensitivity for EHE cosmogenic neutrinos significantly. Previous EHE neutrino searches performed with IceCube 9, 11], showed that IceCube has become the most sensitive neutrino detector in the energy range of $1 \mathrm{PeV}-10 \mathrm{EeV}$ compared to experiments using other techniques [12 16]. The sensitivity of the complete IceCube detector reaches to the modestly high flux cosmogenic models which assume a pure proton composition of cosmic rays. The flux for a heavier composition such as iron is at least 2-3 times lower, although the decrease depends on the source evolution [17] and strongly on the maximal injection energy of the sources [18]. In order to test the heavier composition model predictions, longer exposure or other detection techniques such as the radio detection are needed.

The EHE neutrino search presented here uses data obtained from May 2010 to May 2012. The analysis is sensitive to all three neutrino flavors. The basic search strategies are similar to previous searches $[9,11]$. The main improvement comes from the enlargement of the detector and the statistical enhancement of the data as well as improved modeling of optical properties of the deep glacial ice [19] in the Monte Carlo simulations. The improvements allow a refined geometrical reconstruction of background events and thus a better background rejection. Two neutrino-induced PeV-energy particle shower events were discovered by this EHE neutrino analysis as reported in Ref. [20]. In this paper, we describe the details of the analysis. Then, we investigated whether the two observed events are consistent with cosmogenic neutrinos. Afterwards, cosmogenic neutrino models were tested for compatibility with our observation in order to constrain the UHECR origin.

The paper is structred as follows: in sections [Iand III. the IceCube detector and the data samples are described. The improved analysis methods and the associated systematic uncertainties are discussed in sections [IV] and $[\mathrm{V}$. In section VI results from the analysis are presented. Implications of the observational results on the UHECR origin are discussed in section VII by testing several cosmogenic neutrino models. The model independent upper limit of the EHE neutrino flux is shown in section VIII. Finally, the results are summarized in section IX.

\section{THE ICECUBE DETECTOR}

The IceCube detector observes the Cherenkov light from the relativistic charged particles produced by highenergy neutrino interactions, using an array of Digital Optical Modules (DOMs). Each DOM comprises a 10" R7081-02 photomultiplier tube (PMT) [21] in a transparent pressure sphere along with a high voltage system, a digital readout board 22], and an LED flasher board for optical calibration in ice. These DOMs are deployed along electrical cable bundles that carry power and information between the DOMs and the surface electronics. The cable assemblies, called strings, were lowered into holes drilled to a depth of $2450 \mathrm{~m}$ with an horizontal spacing of approximately $125 \mathrm{~m}$ (Fig. 1). The DOMs sit where the glacial ice is transparent at depths from $1450 \mathrm{~m}$ to $2450 \mathrm{~m}$ at intervals of $17 \mathrm{~m}$. PMT waveforms are recorded when the signal in a DOM crosses a threshold and the nearest or next-to-nearest DOM observes a photon within $1 \mu \mathrm{s}$ (hard local coincidence, HLC). An event is triggered if eight DOMs recorded an HLC within $5 \mu \mathrm{s}$. The lower, inner part of the detector, called DeepCore [23], is filled with DOMs with a smaller vertical and horizontal spacing of $7 \mathrm{~m}$ and $72 \mathrm{~m}$, respectively. The DeepCore array is mainly responsible for the enhancement of the performance below $100 \mathrm{GeV}$, the threshold energy of IceCube. Additional DOMs frozen into tanks located at the surface near the top of each hole constitute an air shower array called IceTop 24]. IceTop allows studying cosmic ray physics and provides the capability to study the atmospheric muon background. The whole detector system comprises 5160 DOMs on 86 strings out of which 8 strings correspond to DeepCore, and an additional 324 DOMs in the surface array. The configurations of the IceCube detectors are displayed in Fig. 1 


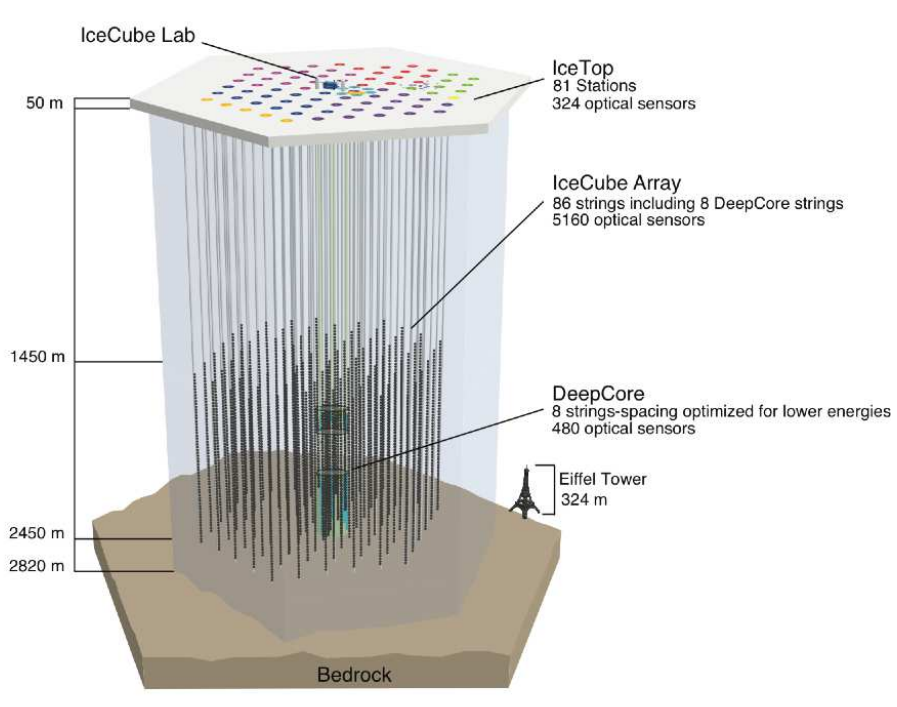

FIG. 1. (color online). A schematic view of the IceCube detector.

\section{DATA AND SIMULATION}

The IceCube detector construction was completed in December, 2010. During the construction phase, from May 31, 2010 to May 12, 2011, 79 strings (IC79), approximately $90 \%$ of the full detector, were operational. The IC79 run was immediately followed by the first year of data taking with the full detector (IC86) which lasted from May 13, 2011 to May 15, 2012. The data from these periods were used in this analysis. The corresponding livetime for the IC79 and IC86 runs are 319.2 days and 350.9 days respectively, excluding periods of detector calibration and unstable operation. Approximately $10 \%$ of the sample (33.4 days of IC79 and 20.8 days of IC86 running) was used as a statistically independent test sample for verification. The final analysis was performed in a blind way where the test sample was not used for the signal search.

There are two classes of background events; atmospheric muon bundle events and events induced by atmospheric neutrinos. Muon bundles consist of a large number of high energy muons produced by cosmic-ray interactions in the atmosphere. Regardless of their high muon multiplicities, they are observed as a single track since their lateral separations of about $10 \mathrm{~m}$ is shorter than the minimum DOM separation of $17 \mathrm{~m}$ except for DeepCore. Since the detector is large and the data recording time window is also long $(10 \mu s)$, there is a non-negligible chance that two or more muon bundles arrive at same time. These events, called "coincident events", complicate geometrical reconstruction. Special treatment is required to reduce this background. Atmospheric muon bundles were simulated with the CORSIKA air-shower simulation 25] with the SIBYLL 2.1 hadronic interaction model [26]. Muons from the showers were propa- gated from the Earth's surface to IceCube depths with the MMC package 27]. These are the same programs as in previous studies [9] except that we have improved our description of the optical properties of the glacier ice [19] used in the simulation of the photon propagation from the particles to the DOMs.

For the atmospheric neutrinos, the All Neutrino Interaction Simulation (ANIS) package [28] is used to simulate each neutrino flavor separately between $50 \mathrm{GeV}$ and $1 \mathrm{EeV}$. The neutrino events were simulated following an $E_{\nu}^{-1}$ spectrum on the surface of the Earth with appropriate flux weights to represent the spectrum resulting from decays of cosmic-ray induced pions and kaons in the atmosphere ("conventional" atmospheric neutrinos). We use the cosmic ray spectrum modeled in Ref. 29] to take into account the spectral bend at the cosmic ray knee. The neutrino multiplicity employed in this calculation is derived from a modified Elbert formula [30, 31]. At PeV energies and above, "prompt" atmospheric neutrinos from decays of charmed mesons are expected to dominate over the conventional atmospheric neutrinos. We consider the default value of the prompt neutrino flux from Enberg et al. [32] modified to incorporate the cosmic-ray spectrum model in Ref. [29].

In order to efficiently simulate high energy events with energies exceeding $100 \mathrm{TeV}$ at IceCube depths, the JULIeT package is used in which the propagation of neutrinos is efficiently obtained by solving numerical transport equations as described in Ref. [8].

Fig. 2 shows examples of simulated signal and background events observed in the IceCube detector. The sizes and colors of the spheres indicate the number and the timing of photo-electrons observed in each DOM. A signal muon event produces a number of stochastic energy losses along the path. Tau events with energies greater than $10 \mathrm{PeV}$ resemble muon tracks, except that they exhibit less energy loss due to their heavier masses. They may also generate characteristic "double bang events" at energies between 1 and $10 \mathrm{PeV}$ due to neutrino interactions and successive tau decays inside the detector volume. Particle showers are induced by neutral current interactions of neutrinos of any flavor or by charged current interactions of electron neutrinos. These events, called cascade events, generate spherical hit patterns in the detector. A background muon bundle event in the current study typically contains about 100 to 1000 muons with lateral separations of about $10 \mathrm{~m}$ which results in a smoother energy loss profile compared to one from a single muon or tau event.

\section{EVENT SELECTIONS}

The energy spectrum of atmospheric muons and neutrinos falls steeply with energy. The cosmogenic neutrino fluxes with their harder spectra are expected to dominate over this background at high energies. Because the amount of deposited energy, i.e. the observable energy, is 


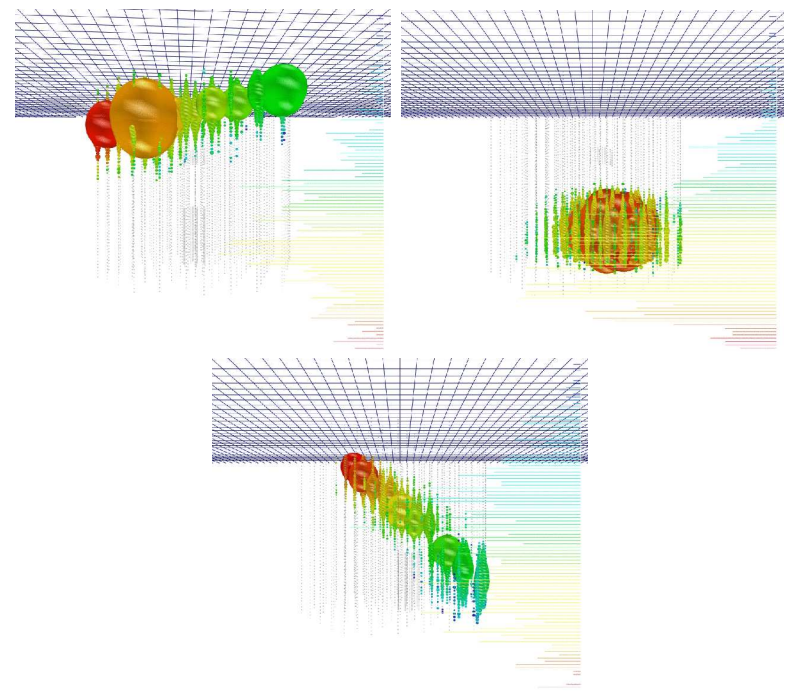

FIG. 2. (color online). Event displays of simulated events. Each sphere represents a DOM. Colors indicate the arrival time of the photon (red indicates the earliest and blue the latest). The size of the sphere and the length of the horizontal lines at the right border indicate the measured amount of photo-electrons in each DOM. Upper left: An upgoing muon entering into the detector array with energy of $20 \mathrm{PeV}$ induced by a neutrino of $500 \mathrm{PeV}$. Upper right: A $300 \mathrm{PeV} \nu_{e}$ induced cascade event. Lower panel: A typical background atmospheric muon bundle event in the current analysis induced by primary cosmic-ray energy of $1 \mathrm{EeV}$.

correlated with the energy of the incoming particles, the signal events stand out against the background events at high energy. Therefore, this analysis is targeted towards the selection of these high energy events.

The initial event filter selects events containing more than 1,000 photo-electrons (p.e.). This filtering eliminates a large number of low-energy atmospheric muon induced events, typically with less than a few $\mathrm{TeV}$ energy. The filtering process is performed at the South Pole and the resulting EHE sample is sent to the North via satellite. The samples contained a total of $4.0 \times 10^{7}$ and $6.0 \times 10^{7}$ events for IC79 and IC86, respectively.

The EHE sample transferred to the North is subjected to off-line hit cleaning in order to remove coincident atmospheric muons and PMT noise. A hit represents a reconstructed pulse of photons from a waveform recorded by a DOM, and is characterized by its time and charge. The initial hit cleaning is a time window cut on the hits outside the time interval between $-4.4 \mu \mathrm{s}$ and $+6.4 \mu \mathrm{s}$ relative to the time of the first hit on the DOM with the highest charge. Then a secondary hit cleaning based on distances and hit time intervals between DOMs is applied. Hits from the DeepCore strings are discarded at this stage and not used for higher selection levels to keep the DOM separation uniform across the detector volume. After these hit cleanings, the analysis level sample was selected by requesting at least 300 hits and 3200 p.e. in the whole detector except DeepCore. This sample con-
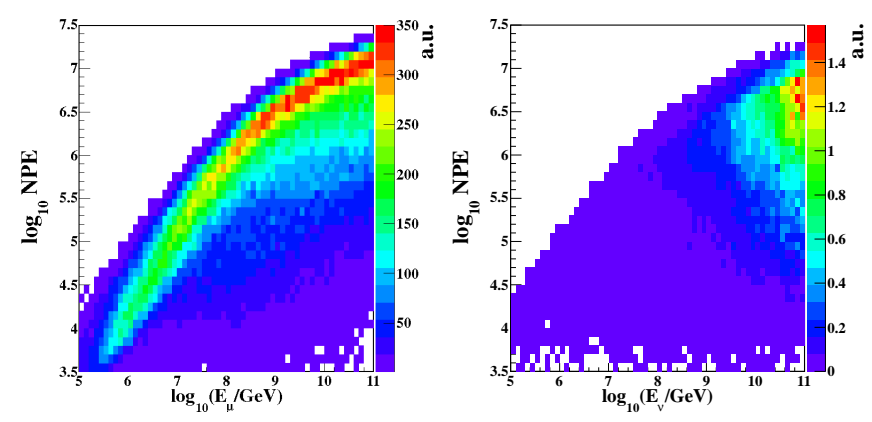

FIG. 3. (color online). Distributions of NPE versus the energies of neutrino-induced muons (left) and neutrinos which induce cascades (right) obtained at the analysis level with the IC86 signal Monte Carlo simulations. For illustrative purposes, an $E^{-1}$ energy spectrum of the particles is assumed in these plots. The muon and neutrino energies are given when the particle enters a radius of $880 \mathrm{~m}$ around the IceCube center. Cascade events include all flavor neutral current and $\nu_{e}$ charged current interactions.

tains a total of $4.5 \times 10^{5}$ and $5.9 \times 10^{5}$ observed events for IC79 and IC86, respectively. The distribution of total number of p.e. (NPE) versus the true energy of the incoming particle for IC86 simulations of neutrino-induced muons and cascades is shown in Fig. 3. The energies are sampled when the incoming particle is at $880 \mathrm{~m}$ from the IceCube center. A clear correlation between NPE and the energy of the muons is observed. By selecting events with NPE above an appropriate threshold, low energy events, dominated by atmospheric backgrounds, are filtered out. The correlation also holds for cascade events although uncontained events with vertex positions outside the instrumentation volume weaken the correlation thereby reducing the selection efficiency for this type of event.

The left panels in Fig. 4 show the NPE distributions at analysis level for data and simulations for IC79 and IC86, respectively. The signal cosmogenic neutrino distributions dominate over the atmospheric $\mu$ and $\nu$ background distributions in the high NPE region. Three cosmogenic neutrino models are shown in the figure: Yoshida et al. [6] for an UHECR source distribution in the form of $(1+z)^{m}$ with the evolution parameter $m=4$ and the maximum redshift of the UHECR source distribution $z_{\max }=4$, Ahlers et al. [33] (the best fit model with $m=4.6$ and $z_{\max }=2.0$ ) and Kotera et al. [17] (Fanaroff-Riley type II). Atmospheric muon bundles are the dominant contribution at this level. Due to the yet unknown chemical composition of UHECRs, the background rates are estimated by the extreme assumptions of pure proton and iron. The pure iron is employed in this analysis as our baseline model for the atmospheric muons since it yields more muons compared to the pure proton case and hence gives us a conservative background estimate. For the pure iron case the predicted rate is about a factor of two higher than the rate observed in IceCube. The data is bracketed by the two compositions as shown in Fig. (4 

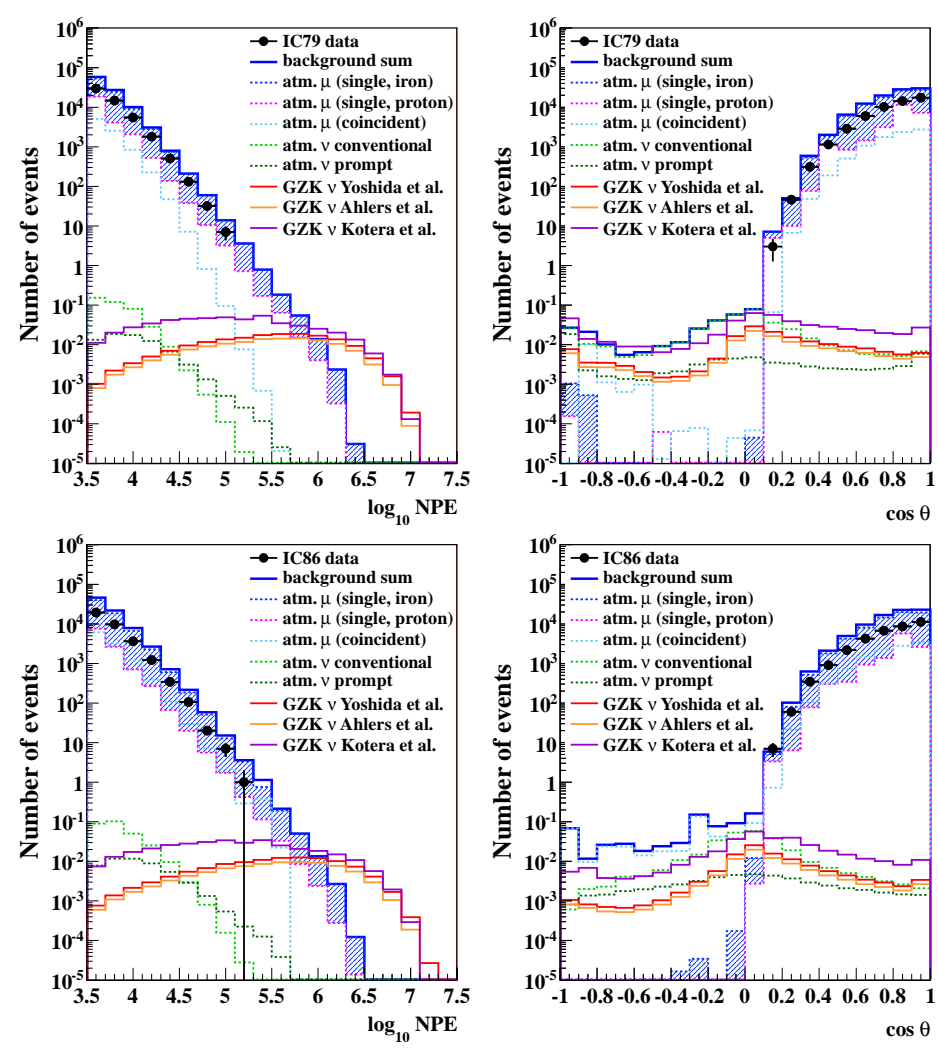

FIG. 4. (color online). Distributions of NPE (left panels) and reconstructed zenith angle (right panels) are shown for the experimental test samples of IC79 (upper panels) and IC86 (lower panels). The data are compared with expected background contributions from atmospheric muons and neutrinos, and signals from various cosmogenic (GZK) neutrino models [6, 17, 33]. The event numbers presented here are for the livetimes of the test samples of the experimental data, 33.4 days for IC79 and 20.8 days for IC86. The signal distributions are the sum of all three neutrino flavors. The background sum includes all atmospheric muons and neutrinos. The single atmospheric muons (pure iron) dominate the background so that the line is nearly identical to the line for the background sum. See text for more detail.

by the shaded area, demonstrating a reasonable agreement between the experimental data and the atmospheric muon background simulations.

The directional information is also used to further discriminate signal from background. Since the background of atmospheric muons is overwhelmingly large compared to our signals above the horizon, a robust directional reconstruction is crucial for the discrimination. For this purpose, a track hypothesis is assumed to reconstruct atmospheric muons. We utilized different zenith angle reconstruction algorithms for IC79 and IC86. A so-called single photo-electron (SPE) Log-Likelihood (LLH) fitting based on a track hypothesis using the probability distribution of the arrival time of the first photon in each DOM [34] is performed for the IC79 sample. Then a cut on the reduced log-likelihood (rLLH) parameter is applied to ensure good fit quality. The parameter rLLH is the log likelihood value of the reconstructed track divided by the number of degrees of freedom of the fit. This rLLH cut removes coincident atmospheric muons. For the IC86 sample, photon hits that have a significantly different timing compared to the one from the main bulk of photon signals are masked using the robust regression technique [35]. Then the particle directions are reconstructed by applying the LineFit algorithm [11] to the remaining unmasked hits. The LineFit algorithm is based on a track hypothesis and uses a simple minimization of $\chi^{2}=\Sigma_{i} \mathrm{NPE}_{i}\left(\vec{r}_{i}-\vec{r}_{\mathrm{COG}}-t_{i} \vec{v}\right)^{2}$, where $t_{i}$ and $\mathrm{NPE}_{i}$ represent the time of the first photo-electron and the number of photo-electrons recorded by the $i$ th DOM at the position $\overrightarrow{r_{i}}$, respectively. The quantity $\vec{r}_{\mathrm{COG}} \equiv\left(\frac{\Sigma_{i} \mathrm{NPE}_{i} x_{i}}{\Sigma_{i} \mathrm{NPE}_{i}}, \frac{\Sigma_{i} \mathrm{NPE}_{i} y_{i}}{\Sigma_{i} \mathrm{NPE}_{i}}, \frac{\Sigma_{i} \mathrm{NPE}_{i} z_{i}}{\Sigma_{i} \mathrm{NPE}_{i}}\right)$ is the position of the NPE-weighted center-of-gravity of the hits. The fit ignores the geometry of the Cherenkov cone and the optical properties of the medium and assumes light traveling with a velocity $\vec{v}$ along a 1 -dimensional path through the detector, passing through the center-of-gravity. The inclusion of the robust regression technique significantly improves the performance of the LineFit used in the previous study [9], allowing for simpler background rejection. The zenith angle resolution of SPE LLH for background muon events is about $0.5^{\circ}$ for the IC79 EHE analysis level sample. The zenith angle resolution from the LineFit with the robust regression for background muons for the IC86 analysis level sample is about $1^{\circ}$. These performances are sufficient to remove atmospheric muon 
bundle background events in the current analysis.

The performance of the reconstruction on the signal neutrinos highly depends on the shape of the events (Fig.2). Since most of the signal neutrino events $(>80 \%)$ are expected to be muon or tau tracks, the reconstruction of zenith angles based upon track hypotheses as described above gives sufficiently good signal selection efficiency. The reconstructed directions of neutrino induced cascades, however, are only poorly correlated with the true neutrino direction and exhibit systematic directional shifts. The SPE LLH reconstruction tends to shift the zenith angles towards the vertical while the LineFit shifts them to the horizontal. The behavior of the shifts also changes when their vertex positions are close to or outside the boundary of the instrumentation volume. The resulting systematic uncertainty is discussed in Section V.

The right panels in Fig. 4 show the event distributions at analysis level as a function of the cosine of the reconstructed zenith angle. These distributions are compared to the background and signal simulations. Atmospheric muon bundles dominate in the downward-going region and atmospheric neutrinos dominate in the upward-going region.

The signal selection criteria were optimized based on simulations of background and signal after the simulation was verified using the test sample. A cosmogenic neutrino model [6] (with $m=4$ and $z_{\max }=4$ ) is used for the optimization. The selection criteria do not severely depend on the particular choice of the cosmogenic model since the expected energy spectrum is similar. Selection criteria are obtained by optimizing the NPE threshold values in the IC79 and IC86 samples separately such that the model discovery factor [9, 37] is minimized in each sample. Fig. 5 presents the event distributions in the plane of NPE vs cosine of the reconstructed zenith angle $(\cos \theta)$ for the test sample and simulations. The distributions of the signal simulation are the sum of all three neutrino flavors. The solid lines in Fig. 5 indicate the final selection criteria for each sample. The events above the lines are considered to be signal event candidates. The essential point of this analysis is to select high NPE events against backgrounds regardless of the event shape. A zenith angle dependent high NPE threshold is required to eliminate the atmospheric muon background for the downward-going region, while a constant threshold value is placed in the zenith region of $\cos \theta \lesssim 0.1$, where no atmospheric muon background is expected. The predicted number of signal and background events passing the final selection criteria are presented in Table 1 along with the observed number of events in the two experimental samples.

The effective neutrino detection areas at final selection criteria for the different IceCube detector configurations are shown in Fig. 6. The effective areas are given for each neutrino flavor, averaged over $4 \pi$ solid angle for IC79 and IC86. The areas are averaged over equal fluxes of neutrinos and anti-neutrinos. Below $5 \mathrm{PeV}$, the effective area for electron neutrinos exceeds that of muon or tau neu- trinos. For particle cascades induced by charged current interactions of electron neutrinos, their energies are deposited completely inside the detector if their interaction vertex lies sufficiently inside the instrumented volume. Contrarily muons (taus) from muon (tau) neutrino interactions only partially deposit their energies in the detector volume. Therefore, even though tracks have a longer path in the detector, they satisfy the NPE criteria less frequently (Fig. 5). At higher energies the effective area for tracks is larger because they can be generated in an increasingly larger volume and still reach the detector. Above $100 \mathrm{PeV}$ cascades contribute less than 20\% to the observable events from cosmogenic neutrino fluxes. The right panel in Fig. 6] shows the effective area summed over all three neutrino flavors for IC79 and IC86 together with that for IC40 from the previous analysis [9]. The current analysis has approximately a factor of two larger effective area compared to IC40. The difference between the effective areas for IC79 and IC86 below $30 \mathrm{PeV}$ originates from the different NPE thresholds. The slight difference above $3 \times 10^{3} \mathrm{PeV}$ is due to the rLLH cut in IC79.

\section{SYSTEMATIC UNCERTAINTIES}

Table II summarizes the statistical and systematic errors for signal, atmospheric muon and neutrino, prompt atmospheric neutrino, and the total background.

One of the dominant sources of systematic uncertainties in the signal event rates is the error associated with the Cherenkov photon measurement, namely the relationship between measured NPE and the energy of the charged particles. This is due to limitations in the understanding of detector sensitivities, photon propagation in the ice and the detector response to bright events which, for example, involves saturation effects of the DOMs. This uncertainty is estimated by calibrating the absolute sensitivity of the DOMs in the laboratory and by measuring it in-situ using a light source co-deployed with the DOMs in the ice [9, 11]. The other uncertainties in the signal rates involve the relevant interactions of neutrinos and leptons produced during the propagation through the Earth. For example, the LandauPomeranchuk-Migdal (LPM) effect [38, 39] can be important since it elongates electromagnetic showers. The elongated shower length is about $20-40 \mathrm{~m}$ for $1-10 \mathrm{EeV}$ electrons [40], thus still being comparable to the IceCube DOM separation of $17 \mathrm{~m}$, and hence negligible. Uncertainties due to other propagation effects are estimated as described in [11].

The uncertainty of the systematic shifts of reconstructed zenith angles for cascade events causes a systematic error in the estimation of the signal neutrino passing rate. The effect is NPE dependent and thus energy dependent. We artificially vary the systematic zenith angle shift by different factors to evaluate the resulting uncertainties. The complete randomization of zenith angles was found to bring the largest reduction of the cas- 
TABLE I. Number of events passing cuts at on-line filtering, off-line analysis, and final level with 285.8 days of effective livetime for IC79 and 330.1 days for IC86 (excluding test sample data). One cosmogenic neutrino model [6] (with $m=4$ and $\left.z_{\max }=4\right)$ is taken to evaluate the benchmark signal rates. The background rates include atmospheric muons assuming a pure iron primary composition, conventional atmospheric neutrinos, and prompt atmospheric neutrinos. Analysis sample requests the number of hit DOMs $\geq 300, \log _{10}(\mathrm{NPE}) \geq 3.5$ for IC79 and IC86, and an additional requirement of rLLH $<8$ for IC79. Systematic uncertainties in the expected event rates at the final selection level are given as asymmetric error intervals after the statistical errors.

\begin{tabular}{|c|c|c|c|c|c|c|}
\hline \multirow{2}{*}{$\begin{array}{l}\text { Contributions } \\
\text { Samples }\end{array}$} & \multicolumn{2}{|c|}{ Experimental } & \multicolumn{2}{|c|}{ Background MC } & \multicolumn{2}{|c|}{ Benchmark signal MC [6] } \\
\hline & IC79 & $\mathrm{IC} 86$ & IC79 & $\mathrm{IC} 86$ & $\mathrm{IC} 79$ & IC86 \\
\hline EHE filter level & $4.0 \times 10^{7}$ & $6.0 \times 10^{7}$ & $4.4 \times 10^{7}$ & $8.9 \times 10^{7}$ & 2.1 & 2.4 \\
\hline Analysis level & $4.5 \times 10^{5}$ & $5.9 \times 10^{5}$ & $8.5 \times 10^{5}$ & $1.3 \times 10^{6}$ & 1.5 & 1.8 \\
\hline Final level & 0 & 2 & $0.056 \pm 0.002_{-0.041}^{+0.028}$ & $0.026 \pm 0.003_{-0.017}^{+0.015}$ & $0.876 \pm 0.004_{-0.105}^{+0.119}$ & $1.043 \pm 0.006_{-0.134}^{+0.142}$ \\
\hline
\end{tabular}
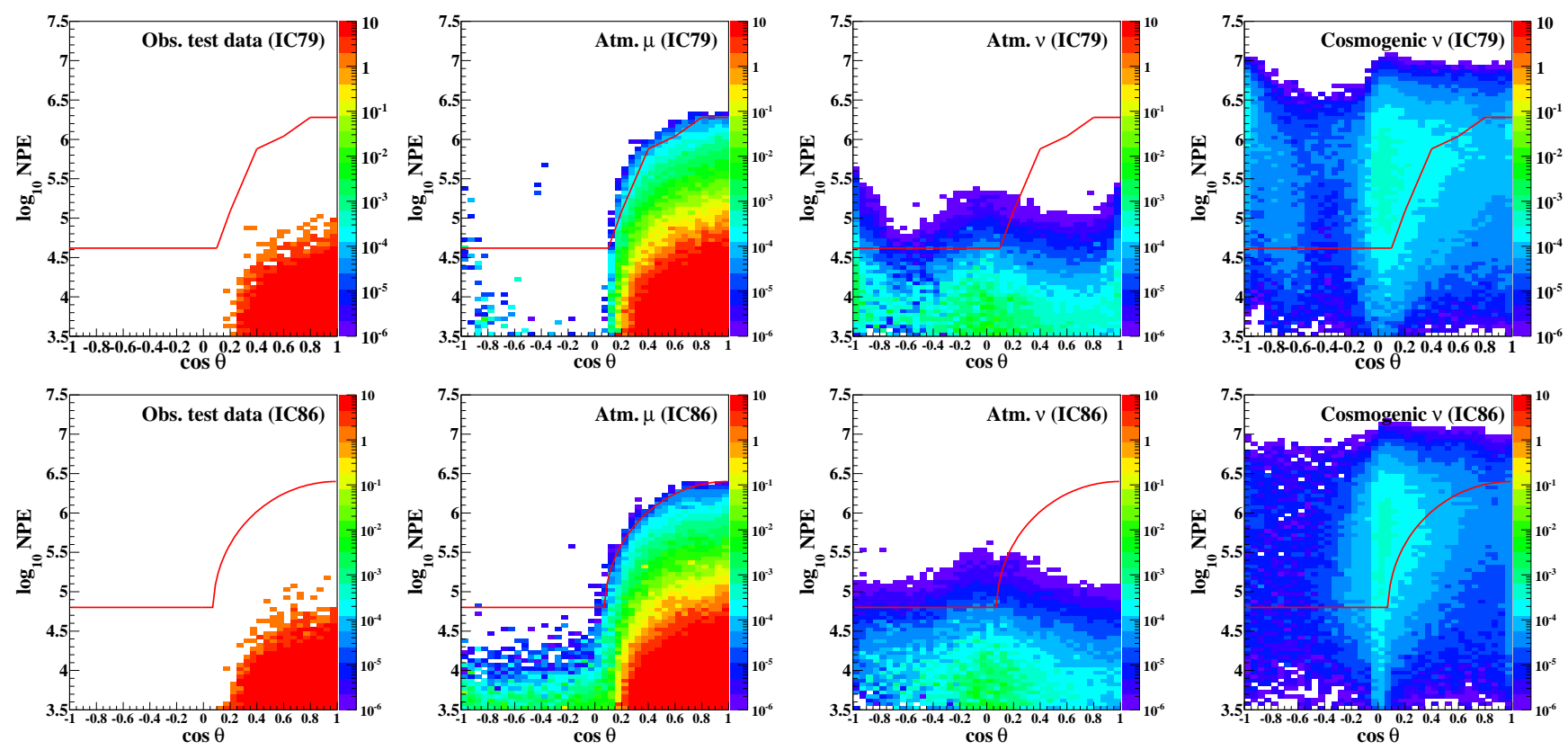

FIG. 5. (color online). Event number distributions on the plane of NPE and cosine of reconstructed zenith angle (cos $\theta$ ) for the IC79 run (upper panels) and the IC86 run (lower panels). The experimental test samples are shown in left panels. The background simulations of atmospheric muon (middle left panels), and the conventional atmospheric neutrino and prompt atmospheric neutrino [32] (middle right panels), and simulation of signal cosmogenic neutrino model [6] (right panels) are also shown. The colors indicate event numbers per livetime of 33.4 days and 20.8 days for the IC79 and IC86 test samples respectively. The signal distributions are the sum of all three neutrino flavors. The solid lines in each panel indicate the final selection criteria.

cade event selection efficiency. The reduction is $20.0 \%$ for events with energies below $10 \mathrm{PeV}, 8.5 \%$ between 10 and $100 \mathrm{PeV}$ and $2.0 \%$ above $100 \mathrm{PeV}$. Since most of the cosmogenic neutrino signal (99.6\%) is expected above $10 \mathrm{PeV}$ and the present analysis is mostly sensitive to track events above $10 \mathrm{PeV}$ as seen in Fig. 6, the effect on the cosmogenic neutrino signal rate is quite limited. The systematic error on the overall signal rate due to the limited performance of the cascade event reconstruction is estimated to be $-0.5 \%$.

Systematic errors in the atmospheric muon background rate arise from uncertainties in the primary cosmic- ray composition, the hadronic interaction model implemented in the air shower simulation, and the cosmicray flux variation at the relevant energies. The two extreme cases of the cosmic-ray compositions, pure iron and pure proton, are used. In the current analysis, the iron-only hypothesis is used for the baseline background rates. This leads to a higher, i.e. conservative, estimate of the photon yield from the muon bundles induced by primary cosmic-ray particles at a particular energy. The difference between the pure-iron and the pure-proton hypothesis then provides the size of the relevant systematic uncertainty. The uncertainty associated with the 

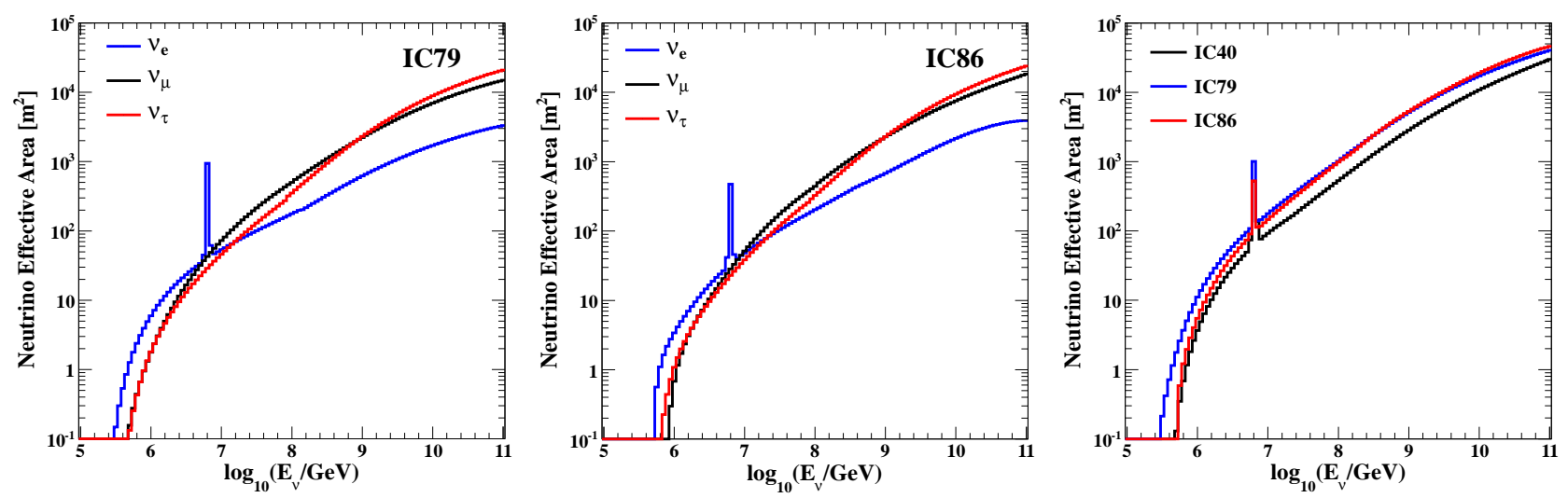

FIG. 6. (color online). The IceCube neutrino effective area at final selection criteria with different string configurations, IC79 (left panel) and IC86 (middle panel) for each neutrino flavor, averaged over $4 \pi$ solid angle. The areas are averaged over equal amounts of neutrinos and anti-neutrinos. Three flavor sums of the effective areas are shown in the right panel. The effective area from the previous search [9] with 40 string configuration of IceCube (IC40) is also shown for comparison. Exposure of the sample used in this analysis is obtained by multiplying the effective area with the effective livetime without test samples (333.5 days, 285.8 days and 330.1 days for IC40, IC79, and IC86 respectively) and $4 \pi$ solid angle. The sharp peaked structure at $6.3 \mathrm{PeV}$ for electron neutrinos is due to the Glashow resonance [36].

TABLE II. List of the statistical and systematic errors on the signal, atmospheric muon and neutrino, prompt neutrino and the total background rate. The uncertainties in the signal rate are estimated for the cosmogenic flux of Yoshida et al. [6] for $\left(m, z_{\max }\right)=(4,4)$. The uncertainties in the background rates are evaluated against the baseline estimation by CORSIKASIBYLL [25, 26] with a pure iron composition hypothesis for atmospheric muons and the Gaisser-H3a model [29] for atmospheric neutrinos. The uncertainties in the prompt neutrino rate are estimated using the prediction by Ref. [32]. The systematic and statistical errors listed here are relative to the event rates for each signal and background source.

\begin{tabular}{|c|c|c|c|c|c|}
\hline Sources & $\begin{array}{l}\text { Cosmogenic } \\
\nu \text { signal }(\%)\end{array}$ & $\begin{array}{c}\text { Atmospheric } \\
\text { muon }(\%)\end{array}$ & $\begin{array}{c}\text { Conventional } \\
\text { atmospheric } \\
\text { neutrino }(\%)\end{array}$ & $\begin{array}{c}\text { Prompt } \\
\text { neutrino }(\%)\end{array}$ & $\begin{array}{c}\text { Total } \\
\text { background }(\%)\end{array}$ \\
\hline Statistical error & \pm 0.4 & \pm 9.1 & \pm 9.8 & \pm 1.1 & \pm 4.5 \\
\hline DOM efficiency & $\begin{array}{l}+1.5 \\
-5.1\end{array}$ & $\begin{array}{l}+41.9 \\
-42.7\end{array}$ & $\begin{array}{l}+73.2 \\
-17.9\end{array}$ & $\begin{array}{l}+33.6 \\
-9.6\end{array}$ & $\begin{array}{l}+43.1 \\
-26.1\end{array}$ \\
\hline Ice properties/Detector response & -7.2 & -47.7 & -44.8 & -30.8 & -41.7 \\
\hline Neutrino cross section & \pm 9.0 & - & - & - & - \\
\hline Photo-nuclear interaction & +10.0 & - & - & - & - \\
\hline LPM effect & \pm 1.0 & - & - & - & - \\
\hline Angular shift for cascades & -0.5 & - & - & - & - \\
\hline Cosmic-ray flux variation & - & $\begin{array}{l}+30.0 \\
-50.0\end{array}$ & \pm 30.0 & \pm 30.0 & $\begin{array}{l}+18.7 \\
-26.3\end{array}$ \\
\hline Cosmic-ray composition & - & -79.1 & - & - & -36.7 \\
\hline Hadronic interaction model & - & +17.7 & - & - & +8.1 \\
\hline$\nu$ yield from cosmic-ray nucleon & - & - & \pm 15.0 & - & \pm 2.2 \\
\hline Prompt model uncertainty & - & - & - & $\begin{array}{l}+31.6 \\
-40.4\end{array}$ & $\begin{array}{l}+12.6 \\
-16.1\end{array}$ \\
\hline \multirow[t]{2}{*}{ Total } & \pm 0.4 (stat.) & \pm 9.1 (stat.) & \pm 9.8 (stat.) & \pm 1.1 (stat.) & \pm 4.5 (stat.) \\
\hline & ${ }_{-12.4}^{+13.6}$ (syst.) & ${ }_{-100}^{+54.5}$ (syst.) & $\begin{array}{l}+80.5 \\
-58.7\end{array}$ (syst.) & ${ }_{-59.8}^{+55.0}$ (syst.) & ${ }_{-68.7}^{+49.3}$ (syst.) \\
\hline
\end{tabular}

hadronic interaction model is estimated by switching the model from SIBYLL 2.1 [26] to QGSJET-II-03 [41] in the simulations. The uncertainty in the cosmic-ray flux normalization is estimated from the variance in the flux measured by several experiments [42, 43] relative to the one used in this analysis 44] at $10 \mathrm{EeV}$, the peak energy of primary cosmic rays that produce atmospheric muon events passing the final selection criteria. The contribu- tion of the cosmic-ray normalization to the uncertainty in the atmospheric neutrino rate is estimated in a similar way at energies from 1 to $100 \mathrm{PeV}$ from various models 29, 45]. In addition, a systematic uncertainty for the atmospheric neutrino rate arises from the uncertainty of the parametrization of the neutrino multiplicity as described in section III A comparison to the full simulation by CORSIKA [25] provides the relevant uncertainty. The 


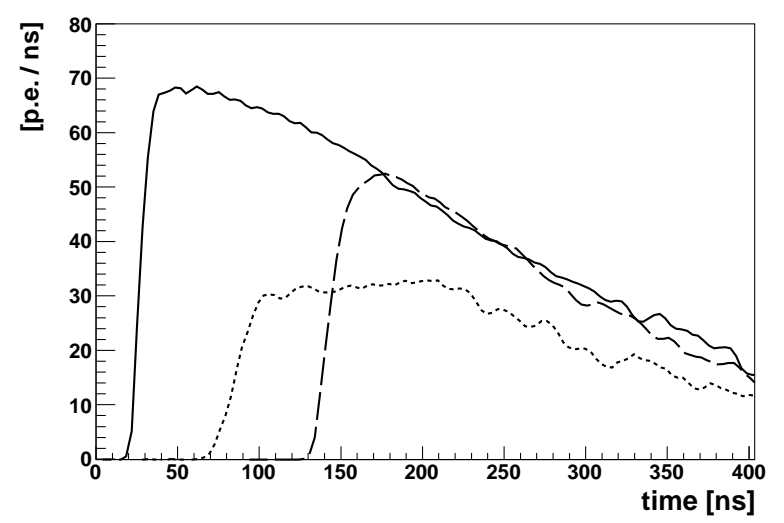

FIG. 7. Waveforms of PMT outputs captured by three DOMs in the neighborhood of the reconstructed vertex position of the event obtained in January, 2012. The waveform drawn as a solid curve is recorded in the DOM closest to the vertex (the brightest DOM). The waveform in the lower (upper) next-tonearest to the brightest DOM on the same string is shown as a dashed (dotted) curve. Photons arrive earlier in the upper DOM because it is closer to the cascade vertex than the lower DOM. The signals from the upper DOM exhibit clear signatures of scattered late photons, suggesting that this cascade is a downward-going event.

systematic uncertainties for backgrounds associated with the photon detection efficiency and the optical properties of the ice are determined in the same manner as for signal events.

The atmospheric neutrino background is calculated over $4 \pi$ solid angle and simulated independently of the atmospheric muon background. In reality, downwardgoing atmospheric neutrino events would be accompanied by atmospheric muons, which improves their geometrical reconstruction. Because correctly reconstructed downward-going events are mostly rejected due to the higher NPE threshold employed in the final event selection, the background rate obtained from the independent neutrino and muon simulations is likely overestimated.

The systematic error on the prompt neutrino flux is estimated similarly. Relatively large uncertainty arise from the parametrization in the framework of the Enberg el al. model [32] which we used for the calculation of the baseline rate of prompt neutrinos. A possible nonperturbative QCD contribution in charm production involves an even larger uncertainty. We have not observed clear evidence for prompt contributions in atmospheric neutrinos so far [46].

\section{RESULTS}

Two events passing the final selection criteria are observed [20]. The waveform profiles and the detector hit patterns of both events are consistent with that of Cherenkov photons from particle cascades induced by neutrinos well inside the IceCube instrumentation vol-
TABLE III. The 90\% C.L. of the energy range of the primary neutrino in $\mathrm{PeV}$ at the Earth's surface for the two events for an energy spectrum following an $\mathrm{E}^{-2}$ power law.

\begin{tabular}{lc}
\hline \hline & Energy range (90\% C.L.) \\
\hline \hline Event (August, 2011) & $0.81-7.6 \mathrm{PeV}$ \\
Event (January, 2012) & $0.93-8.9 \mathrm{PeV}$ \\
\hline \hline
\end{tabular}

ume. There is no indication of outgoing/incoming muon or tau tracks. Several waveforms captured by the DOMs in the neighborhood of one of the reconstructed cascade vertex position are shown in Fig. 7 . The total charge contained in the waveforms plays a dominant role in estimating the deposited energy of the cascade. The leading edge time mainly determines the vertex position. The relative widths of the waveforms in DOMs in the forward an rear direction of the cascade is relevant for the reconstruction of the arrival direction of neutrinos. Since photons can only reach the backward direction by scattering, the distribution of photon arrival times is much wider in the backward region of the cascades. The relations of the waveform features to the energy, direction, and vertex position are described using a single likelihood function built from a product of Poisson probabilities of the number of photons predicted to arrive in a given time bin against the number extracted from the recorded waveform. Minimizing the log likelihood under simultaneous variation of the energy and geometry of the cascade hypothesis yields estimates of the deposited energy, direction, and interaction vertex of the cascade.

The reconstructed deposited energies of the two observed cascades are $1.04 \pm 0.16 \mathrm{PeV}$ and $1.14 \pm 0.17 \mathrm{PeV}$, respectively. The statistical energy resolutions for these events are obtained by simulating cascades with parameters close to the reconstructed energies and cascade vertices, and are found to be $3 \%$. The total error on the energy is dominated by systematic uncertainties. These include the absolute detection efficiency of the DOM and the optical properties of the ice, both of which are major factors when relating the number of observed photons to the cascade energy. The size of the errors are estimated by reconstructing simulated events with various models of the ice properties.

The incoming neutrino energy corresponds exactly to the deposited cascade energy if a charged-current interaction of an electron neutrino induces a cascade. For neutral current reactions of neutrinos of any flavor, only a fraction of the neutrino energy is transferred to a cascade depending on the inelasticity of the collision. Because the present analysis is incapable of distinguishing between neutrino flavors, both interaction channels are included when constructing the probability density function (PDF) of the energy of the incoming neutrino. Here, the systematic uncertainties for the deposited energies are taken into account. The PDF of the neutrino en- 
ergy at the surface of the Earth is built by simulating neutrino interactions over a wide energy range each time evaluating the probability that the resulting cascade energy matches the estimated energy and its uncertainty. The $90 \%$ C.L. energy ranges obtained from the PDFs for neutrino spectra with an $E_{\nu}^{-2}$ power law flux are summarized in Table III The flavor ratio is assumed to be $\nu_{e}: \nu_{\mu}: \nu_{\tau}=1: 1: 1$. Since the neutrino-nucleon interaction cross section increases with neutrino energy, the possibility that the energy of the primary neutrino is much higher than the observed cascade energy is not entirely negligible, depending on the neutrino spectrum. For example, the $90 \%$ C.L. energy range for a cosmogenic neutrino model [33] extends to about $500 \mathrm{PeV}$, which shows that the energy range heavily depends on the shape of the energy spectrum.

\section{TESTS ON COSMOGENIC NEUTRINO MODELS}

Our results are characterized by two observational facts: the detection of two neutrinos with deposited energies of about one $\mathrm{PeV}$ and the non-detection of neutrinos with higher deposited energies. First, we investigate whether a single cosmogenic neutrino model can account for these two observational facts simultaneously. Secondly, we constrain the UHECR origin with the present results. Because most cosmogenic neutrinos have energies above $100 \mathrm{PeV}$, tests on the event rate above this energy expected from cosmogenic neutrino models under various assumptions on the UHECR spectrum and the evolutions of the source distributions will lead to constrains on the UHECR origin. We note that the energy threshold of $100 \mathrm{PeV}$ is an a posteriori parameter and, hence, the results are not part of the blind analysis.

The statistical significance of these tests is limited by our observational exposure. To obtain the best constraints, we combine the exposure of the previously published results obtained by the half-completed IceCube detector with its 40 string configuration (IC40) [9] with the present results hereafter. The IC40 data increases the observational exposure by about $30 \%$, depending on the neutrino energy, as displayed in Fig. 6 .

\section{A. The full energy range test}

We introduce here an energy inclusive test which checks the consistency of the energy distributions of cosmogenic neutrino models with the observed two events. A p-value is calculated with the Kolmogorov-Smirnov test (KS-test) using the energy spectrum of the neutrino models and the energy PDFs of the two observed events. The expected energy distributions from the neutrino models are obtained by multiplication of the neutrino effective area with the predicted neutrino energy spectrum. This allows us to analytically calculate p-
TABLE IV. P-values $P_{\mathrm{E}}$ in Eq. 1 are listed for several neutrino models. All the models shown here assume the cosmic-ray primaries to be protons and different spectral indices/cut-off energies at sources, IR/UV backgrounds, as well as different cosmological evolution parameters and extension in redshift for the sources. P-values for $E_{\nu}^{-2}$ spectra with various cut-off energies are also shown for reference.

\begin{tabular}{lc}
\hline \hline$\nu$ Model & p-value \\
\hline Yoshida and Teshima [6] \\
$m=4.0, z_{\max }=4.0$ & 0.077 \\
\hline $\begin{array}{l}\text { Ahlers } \text { et al. }[33] \\
\text { Kotera } \text { et al. }[17]\end{array}$ & \\
GRB & 0.052 \\
\hline Kotera et al. [17] & \\
Fanaroff-Riley type II & 0.039 \\
\hline$E_{\nu}^{-2}$ & 0.18 \\
with cut-off at $10 \mathrm{PeV}$ & 0.13 \\
with cut-off at $100 \mathrm{PeV}$ & 0.11 \\
with cut-off at $1 \mathrm{EeV}$ & \\
\hline \hline
\end{tabular}

values without relying on extensive Monte-Carlo simulations. In order to evaluate the final p-value, $P_{E}$, that the two events (energies $E_{1}$ and $E_{2}$ ) are consistent with a cosmogenic flux model, the p-value obtained in the $\mathrm{KS}$ test, $P_{\mathrm{KS}}\left(E_{1}, E_{2}\right)$, is convoluted with the energy PDFs of the two events as follows:

$$
P_{\mathrm{E}}=\int d E_{1} \rho_{1}\left(E_{1}\right) \int d E_{2} \rho_{2}\left(E_{2}\right) P_{\mathrm{KS}}\left(E_{1}, E_{2}\right),
$$

where $\rho_{i}$ is the energy PDF of the $i$ th event. Note that the PDF is different for each model to be tested as described in the previous section. Table IV summarizes the resulting p-values of this test: all cosmogenic neutrino models are inconsistent with the two observed events at more than $90 \%$ C.L.

The recent follow-up analysis [47] revealed the existence of neutrinos at $\mathrm{TeV}$ energies above the atmospheric background, in addition to the two $\mathrm{PeV}$ events reported in Ref. [20]. The event distribution indicated either a substantially softer spectrum than $E_{\nu}^{-2}$ or the presence of a break or cut-off at PeV energies, although the statistics are limited. The present analysis confirmed this picture using the KS test with an $E_{\nu}^{-2}$ spectrum hypothesis as Table IV lists the resultant p-values with various assumptions of the spectral cut-off energies. The observed $\mathrm{PeV}$ events are unlikely to originate from a bulk of neutrinos with energies extending well above $\mathrm{PeV}$, regardless of the characteristics of the events at $\mathrm{TeV}$ energies found in the follow-up analysis.

\section{B. The ex post facto test above $100 \mathrm{PeV}$}

Here, a prospective event rate in the energy region above $100 \mathrm{PeV}$ is compared to the observational upper 
limit. A constraint on a given neutrino model is set by calculating the model rejection factor (MRF) [48] given by

$$
R_{\mathrm{MRF}}=\frac{N_{100(1-\alpha) \%}}{\mu_{\nu}},
$$

where $N_{100(1-\alpha) \%}$ is the upper limit of number of events at $100(1-\alpha) \%$ C.L. and $\mu_{\nu}$ is the event rate of signal neutrino events predicted by the model above $100 \mathrm{PeV}$. Any model with $R_{\mathrm{MRF}} \leq 1$ is rejected at $\geq 100(1-\alpha) \%$ C.L. in this approach. For $\alpha=0.1, N_{90 \%}=2.27$ in the Feldman-Cousins approach [49] for a null observation with a conventional background of 0.16 events. The large number of background events comes mainly from the IC40 analysis contributing 0.11 event [9]. Although the probability that the original neutrino energy of the two observed events is higher than $100 \mathrm{PeV}$ is expected to be small, this is taken into account by calculating the most probable upper limit:

$$
N_{100(1-\alpha) \%}=\sum_{n=0}^{2} P_{n} N_{100(1-\alpha) \%}^{n} .
$$

Here, $P_{n}$ is the probability of finding $n$ events above $100 \mathrm{PeV}$ determined by the energy PDFs of the two events, and $N_{100(1-\alpha) \%}^{n}$ is the upper limit for $n$ observed events. Since the energy PDF highly depends on the shape of the energy spectrum, an appropriate shape of an energy spectrum has to be chosen. Since the two observed events were found to be inconsistent with cosmogenic neutrino models as shown in the previous subsection, the cosmogenic neutrino models are not used for the energy PDF, instead an $E^{-2}$ power law spectrum is used. The $N_{90 \%}$ is calculated for the standard cosmogenic models, and found to be 2.273 which is slightly larger than for the case of a null detection. The systematic uncertainty on the background estimates is incorporated using a method outlined in [50]. The p-value $\alpha$ for a given model is obtained by requesting $R_{\mathrm{MRF}}=1$ in Eq. 2.

Table $\mathrm{V}]$ summarizes the p-values for several neutrino models. The maximal flux allowed by the constraints from the diffuse photon flux (labeled as "the maximal flux" in the table) is excluded at 95\% C.L. It demonstrates that the present constraints from the limit on the ultra-high energy neutrino flux are compatible with those from photon flux measurements by Fermi in the $10 \mathrm{GeV}$ region [55].

In order to set constraints on characteristics of the UHECR sources in a more comprehensive manner, a parametrization often used in the literature [6] is employed, in which the spectral emission rate per co-moving volume scales as $(1+z)^{m}$ for $z \leq z_{\max }$. The event rate at energies above $100 \mathrm{PeV}$ is calculated for a given $m$, and $z_{\max }$ using the formula in Ref. [4]. The constraints on the parameter space of $m$ and $z_{\max }$ are derived by using Eq. 2, and are displayed in Fig. 8]
TABLE V. Expected numbers of events from several neutrino models and the p-values for consistency with the present ob-

\begin{tabular}{|c|c|c|}
\hline$\nu$ Model & Event rate above $100 \mathrm{PeV}$ & p-value \\
\hline $\begin{array}{l}\text { Yoshida and Teshima [6] } \\
m=4.0, z_{\max }=4.0\end{array}$ & 2.0 & 0.14 \\
\hline $\begin{array}{l}\text { Kalashev et al. }[51] \\
m=5.0, z_{\max }=3.0\end{array}$ & 3.1 & 0.045 \\
\hline $\begin{array}{l}\text { Yoshida and Ishihara }[4] \\
m=5.0, z_{\max }=2.0\end{array}$ & 1.5 & 0.22 \\
\hline $\begin{array}{l}\text { Ahlers et al. }[33] \\
m=4.6, z_{\max }=2.0 \\
\text { ("the best fit") }\end{array}$ & 1.5 & 0.22 \\
\hline $\begin{array}{l}\text { Ahlers et al. } 33] \\
\text { ("the maximal flux") }\end{array}$ & 3.1 & 0.044 \\
\hline $\begin{array}{l}\text { Kotera et al. } 17] \\
\text { GRB }\end{array}$ & 0.48 & 0.66 \\
\hline $\begin{array}{l}\text { Kotera et al. [17] } \\
\text { SFR }\end{array}$ & 0.46 & 0.67 \\
\hline $\begin{array}{l}\text { Kotera et al. }[17] \\
\text { Fanaroff-Riley type II }\end{array}$ & 2.9 & 0.052 \\
\hline $\begin{array}{l}\text { Top-down } 1[52] \\
\text { SUSY }\end{array}$ & 16 & $\leq 0.0020$ \\
\hline $\begin{array}{l}\text { Top-down } 2[52] \\
\text { GUT }\end{array}$ & 3.9 & 0.021 \\
\hline
\end{tabular}
servation in energy range above $100 \mathrm{PeV}$.

\section{Discussion}

The models listed in the top two rows of Table IV assume that the ankle structure which appears at 3 to $10 \mathrm{EeV}$ in the UHECR spectrum is due to the transition from the Galactic to the extragalactic component [56]. In this scenario, the cosmogenic neutrino generation mechanism is dominated by collisions of UHECRs with the CMB photons which results in a neutrino energy spectrum with a peak at about $1 \mathrm{EeV}$, well above the main regime of the energy range of the two observed events. This is the reason why these models are inconsistent with the two observed events as shown in Table IV. The models in the lower two rows of Table IV (Kotera et al. [17]) assume the "dip" transition model [57] where the ankle structure is mainly caused by pair-production energy losses of UHECRs on diffuse infrared, optical, and ultraviolet backgrounds (IR/UV backgrounds) during intergalactic propagation. The neutrino models in Kotera et al. use the IR/UV backgrounds as modeled by Stecker [58] which comprises an increased far-infrared bump at large redshift (note that the IR/UV model employed in these neutrino models is now disfavored by gamma-ray observation with Fermi-LAT [59]). Compared to the standard cosmogenic models, the dip and the IR/UV backgrounds leads to an increased flux of neutrinos at $\mathrm{PeV}$ energies, so that these models in Kotera et al. could be more consistent with the observation. However, even in these models, the collision of UHECRs with 


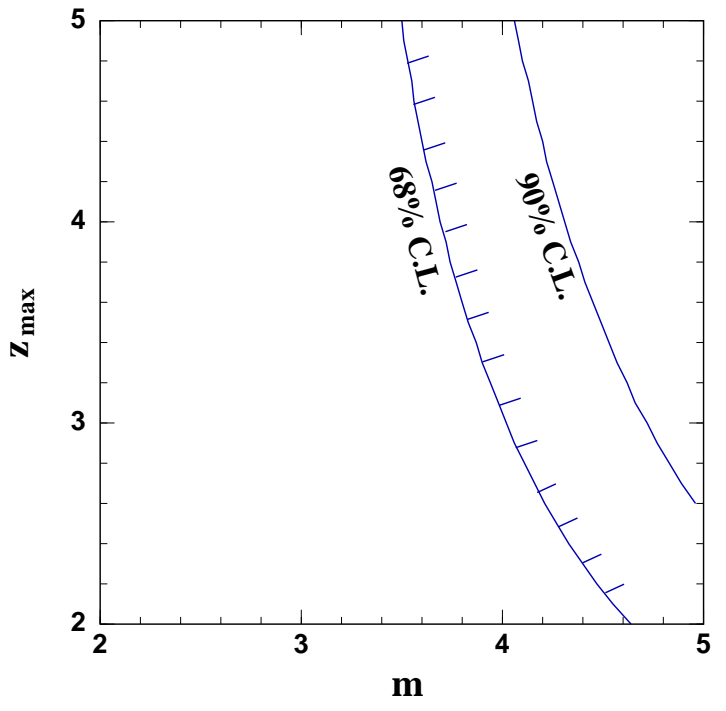

FIG. 8. Constraints on the UHECR source evolution parameters of $m$ and $z_{\max }$ with the present analysis. The semianalytic formulation [4] estimates the neutrino flux for calculating the limit shown here. The area above the solid lines is excluded at the quoted confidence level.

CMB photons produces a bulk of neutrinos with energies much higher than $100 \mathrm{PeV}$ which should have been detected because of the significantly larger effective area at these energies. In addition, the substantial flux at $\mathrm{PeV}$ energies yields energy PDFs for the observed two events very similar to those from an $E_{\nu}^{-2}$ spectrum. Since the energy range for the $E_{\nu}^{-2}$ spectrum PDF does not extend to $10 \mathrm{PeV}$ as shown in Table III neutrinos with energy of $100 \mathrm{PeV}$ or greater are less likely to be responsible for the observed PeV cascades. Because of these reasons, p-values for these scenarios in Kotera et al. are small as shown in Table IV] In conclusion, none of the cosmogenic scenarios is consistent with the observation of the two events. This indicates that models which predict neutrino spectra extending to energies well beyond 100 $\mathrm{PeV}$ will not explain our measurements.

The model test based on the event rates above $100 \mathrm{PeV}$ indicates that strong source evolution models $(m \gg 4)$ are not responsible for the bulk of UHECRs. Among sources categorized in this class are the Fanaroff-Riley type II (FR-II) radio galaxies, the long-standing favorite as a candidate of the UHECR emitters [60]. Similarly a strong source evolution model for GRBs [61] is also rejected by our observation since the model produces higher neutrino flux than the FR-II model. The obtained limits are highly complementary to the bound from the diffuse photon flux [55], because the cosmogenic neutrino intensity around $1 \mathrm{EeV}$, the central energy range of the presented search with IceCube, is stable against uncertainties in the IR/UV backgrounds and the transition model between the galactic and extragalactic component of the UHECRs [4, 17, 62, 63]. We should note, however, that the obtained bound is not valid if the mass compo-

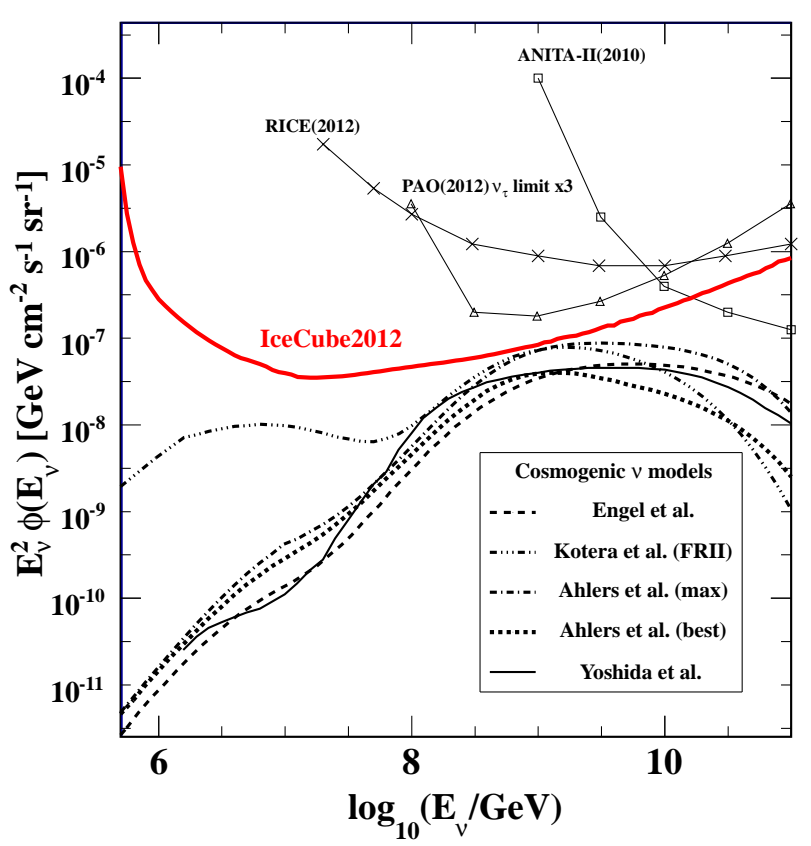

FIG. 9. All flavor neutrino flux differential 90\% C.L. upper limit evaluated for each energy with a sliding window of one energy decade from the present IceCube EHE analysis including the IceCube exposure from the previously published result (IC40) [9]. All the systematic errors are included. Various model predictions (assuming primary protons) are shown for comparison; Engel et al. 7], Kotera et al. [17], Ahlers et al. [33], Yoshida et al. [6]. The model-independent differential $90 \%$ C.L. upper limits for one energy decade by other experiments are also shown for Auger (PAO) [53], RICE [54], ANITA [14, 15] with appropriate normalization by taking into account the energy bin width and the neutrino flavor. The upper limit for the $\nu_{\tau}$ flux obtained by Auger is multiplied by three to convert it to an all flavor neutrino flux limit (assuming an equal neutrino flavor ratio).

sition of UHECRs is not dominated by proton primaries. The dominance of proton primaries is widely assumed in the models mentioned here while a dominance of heavier nuclei such as iron provides at least 2-3 times lower neutrino fluxes. The analysis is not sensitive enough to reach these fluxes yet.

\section{THE MODEL INDEPENDENT UPPER LIMIT}

The quasi-differential, model-independent 90\% C.L. upper limit on all flavor neutrino fluxes, $\phi_{\nu_{e}+\nu_{\mu}+\nu_{\tau}}$, was evaluated for each energy with a sliding window of one energy decade. It is shown in Fig. 9 using the same method as implemented in our previous EHE neutrino searches [9, 11]. An equal flavor ratio of $\nu_{e}: \nu_{\mu}: \nu_{\tau}=$ $1: 1: 1$ is assumed here. A difference from the calculation of the limit shown in our previous publications arises from the existence of two events in the final sample. The 
$90 \%$ event upper limit used in the calculation takes into account the energy PDFs of each of the observed events using Eq. 3. where $P_{n}$ is a function of the neutrino energy $E_{\nu}$ and corresponds to the probability of having $n$ events in the interval $\left[\log _{10}\left(E_{\nu} / \mathrm{GeV}\right)-0.5, \log _{10}\left(E_{\nu} / \mathrm{GeV}\right)+\right.$ 0.5]. Here, the PDFs for an $E_{\nu}^{-2}$ spectrum are used since the two observed events are not consistent with a harder spectrum such as from cosmogenic neutrino models. The quasi-differential limit takes into account all the systematic uncertainties described in section $\mathrm{V}$. The effect of the uncertainty due to the angular shift of the cascade events on the upper limit is negligible above $10 \mathrm{PeV}(<1 \%)$ as track events dominate in this energy range. Below $10 \mathrm{PeV}$, the effect weakens the upper limit by $17 \%$ because cascade events dominate. Other systematic uncertainties are implemented as in previous EHE neutrino searches [9, 11]. The obtained upper limit is the strongest constraint in the EeV regime so far. In the $\mathrm{PeV}$ region, the constraint is weaker due to the detection of the two events. An upper limit for an $E^{-2}$ spectrum that takes into account the two observed events was also derived and amounts to $E^{2} \phi_{\nu_{e}+\nu_{\mu}+\nu_{\tau}}=2.5 \times 10^{-8} \mathrm{GeV} \mathrm{cm}^{-2}$ $\mathrm{s}^{-1} \mathrm{sr}^{-1}$ for an energy range of $1.6 \mathrm{PeV}-3.5 \mathrm{EeV}(90 \%$ event coverage).

\section{SUMMARY}

We analyzed the 2010-12 data samples collected by the 79 and 86-string IceCube detector searching for extremely high energy neutrinos with energies exceeding $1 \mathrm{PeV}$. We observed two neutrino-induced cascade events passing the final selection criteria. The energy profiles of the two events indicate that these events are cascades with deposited energies of about $1 \mathrm{PeV}$. The cosmogenic neutrino production is unlikely to be responsible for these events. An upper limit on the neutrino rate in the energy region above $100 \mathrm{PeV}$ places constraints on the redshift distribution of UHECR sources. For the first time the observational constraints reach the flux region pre- dicted for some UHECR source class candidates. The obtained upper limit is significantly stronger compared to our previous publication [9] because of the enlarged instrumented volume and the refined Monte Carlo simulations. Future data obtained with the completed detector will further enhance IceCube's sensitivity to cosmogenic neutrino models.

\section{ACKNOWLEDGMENTS}

We acknowledge the support from the following agencies: U.S. National Science Foundation-Office of Polar Programs, U.S. National Science FoundationPhysics Division, University of Wisconsin Alumni Research Foundation, the Grid Laboratory Of Wisconsin (GLOW) grid infrastructure at the University of Wisconsin - Madison, the Open Science Grid (OSG) grid infrastructure; U.S. Department of Energy, and National Energy Research Scientific Computing Center, the Louisiana Optical Network Initiative (LONI) grid computing resources; Natural Sciences and Engineering Research Council of Canada, WestGrid and Compute/Calcul Canada; Swedish Research Council, Swedish Polar Research Secretariat, Swedish National Infrastructure for Computing (SNIC), and Knut and Alice Wallenberg Foundation, Sweden; German Ministry for Education and Research (BMBF), Deutsche Forschungsgemeinschaft (DFG), Helmholtz Alliance for Astroparticle Physics (HAP), Research Department of Plasmas with Complex Interactions (Bochum), Germany; Fund for Scientific Research (FNRS-FWO), FWO Odysseus programme, Flanders Institute to encourage scientific and technological research in industry (IWT), Belgian Federal Science Policy Office (Belspo); University of Oxford, United Kingdom; Marsden Fund, New Zealand; Australian Research Council; Japan Society for Promotion of Science (JSPS); the Swiss National Science Foundation (SNSF), Switzerland; National Research Foundation of Korea (NRF)
[1] K. Greisen, Phys. Rev. Lett. 16, 748 (1966).

[2] G. Zatsepin and V. Kuzmin, JETP Lett. 4, 78 (1966).

[3] V. Berezinsky and G. Zatsepin, Phys. Lett. B 28, 423 (1969).

[4] S. Yoshida and A. Ishihara, Phys.Rev. D 85, 063002 (2012).

[5] M. Ahlers, L. A. Anchordoqui, and S. Sarkar, Phys. Rev. D 79, 083009 (2009).

[6] S. Yoshida and M. Teshima, Prog. Theor. Phys. 89, 833 (1993)

[7] R. Engel, D. Seckel, and T. Stanev, Phys. Rev. D 64, 093010 (2001).

[8] S. Yoshida, R. Ishibashi and H. Miyamoto, Phys. Rev. D 69, 103004 (2004).

[9] R. Abbasi et al. (IceCube Collaboration), Phys. Rev. D 83, 092003 (2011).
[10] A. Achterberg et al. (IceCube Collaboration), Astropart. Phys. 26, 155 (2006).

[11] R. Abbasi et al. (IceCube Collaboration), Phys. Rev. D 82, 072003 (2010).

[12] J. Abraham et al. (Pierre Auger Collaboration), Phys. Rev. D 79, 102001 (2009).

[13] P. Abreu et al. (Pierre Auger Collaboration), Phys. Rev. D 84, 122005 (2011).

[14] P. W. Gorham et al. (ANITA Collaboration), Phys. Rev. D 82, 022004 (2010).

[15] P. W. Gorham et al. (ANITA Collaboration), Phys. Rev. D 85, 049901(E) (2012).

[16] I. Kravchenko et al., Phys. Rev. D 73, 082002 (2006).

[17] K. Kotera, D. Allard, and A. Olinto, JCAP 1010, 013 (2010). 
[18] M. Ahlers and F. Halzen, Phys. Rev. D 86, 083010 (2012).

[19] M. Aartsen et al. (IceCube Collaboration), Nucl. Instrum. Meth. A 711, 73 (2013).

[20] M. Aartsen et al. (IceCube Collaboration), Phys. Rev. Lett. 111, 021103 (2013).

[21] R. Abbasi et al. (IceCube Collaboration), Nucl. Instrum. Meth. A 618, 139 (2010).

[22] R. Abbasi et al. (IceCube Collaboration), Nucl. Instrum. Meth. A 601, 294 (2009).

[23] R. Abbasi et al. (IceCube Collaboration), Astropart. Phys. 35, 615 (2012).

[24] R. Abbasi et al. (IceCube Collaboration), Nucl. Instrum. Meth. A 700, 188 (2013).

[25] D. Heck et al., CORSIKA: A Monte Carlo code to simulate extensive air showers, FZKA-6019, (1998).

[26] E. J. Ahn, R. Engel, T. K. Gaisser, P. Lipari and T. Stanev, Phys. Rev. D 80, 094003 (2009).

[27] D. Chirkin and W. Rhode, Propagating Leptons through matter with Muon Monte Carlo (MMC), arXiv:hep-ph/0407075 2 .

[28] A. Gazizov and M. P. Kowalski, Comput. Phys. Commun. 172, 203 (2005).

[29] T. K. Gaisser, Astropart. Phys. 35, 801 (2012).

[30] T. K. Gaisser, Cosmic Rays and Particle Physics (Cambridge University Press, England, 1990).

[31] J. Elbert, in Proceedings of the DUMAND Summer Workshop (Scripps Institution of Oceanography, La Jolla, CA, 1979), Vol. 2, p. 101.

[32] R. Enberg, M. H. Reno, and I. Sarcevic, Phys. Rev. D 78, 043005 (2008).

[33] M. Ahlers et al., Astropart. Phys. 34, 106 (2010).

[34] J. Ahrens et al. (AMANDA Collaboration), Nucl. Instrum. Meth. A 524, 169 (2004).

[35] M. G. Aartsen et al. (IceCube Collaboration), arXiv:1308.5501 [astro-ph.IM]; submitted to Nucl. Instrum. Meth. A.

[36] S. L. Glashow, Physical Review 118, 316 (1960).

[37] G. C. Hill et al., in the Proceedings of PHYSTAT2005, Oxford, England, 108 (2006).

[38] L. D. Landau and I. J. Pomeranchuk, Dokl. Akad. Nauk. SSSR 92, 535 (1953).

[39] A. B. Migdal, Physical Review 103, 1811 (1956).

[40] L. Gerhardt and S. R. Klein, Phys. Rev. D 82, 074017 (2010).
[41] S. Ostapchenko, Phys. Rev. D 83, 014018 (2011).

[42] J. Abraham et al. (Pierre Auger Collaboration), Phys. Lett. B 685, 239 (2010).

[43] R. Abbasi et al. (HiRes Collaboration), Phys. Rev. Lett. 100, 101101 (2008).

[44] M. Nagano and A. A. Watson, Rev. Mod. Phys. 72, 689 (2000).

[45] V. Zatsepin and N. V. Sokolskaya, Astron. Astrophys. 458, 1 (2006).

[46] A. Schukraft, Nucl. Phys. Proc. Suppl. 237-238, 266 (2013).

[47] M. Aartsen et al. (IceCube Collaboration), Science 342, 1242856 (2013).

[48] G. Hill and K. Rawlins, Astropart. Phys. 19, 393 (2003).

[49] G. J. Feldman and R. D. Cousins, Phys. Rev. D 57, 3873 (1998).

[50] F. Tegenfeldt and J. Conrad, Nucl. Instrum. Methods Phys. Res., Sect. A 539, 407 (2005).

[51] O. E. Kalashev, V. A. Kuzmin, D. V. Semikoz, and G. Sigl, Phys. Rev. D 66, 063004 (2002).

[52] G. Sigl, S. Lee, P. Bhattacharjee, and S. Yoshida, Phys. Rev. D 59, 043504 (1998).

[53] P. Abreu et al. (Pierre Auger Collaboration), Astrophys. J. 755, L4 (2012).

[54] I. Kravchenko et al., Phys. Rev. D 85, 062004 (2012).

[55] V. Berezinsky, A. Gazizov, M. Kachelriess, and S. Ostapchenko, Phys. Lett. B 695, 13 (2011).

[56] T. Wibig and A. W. Wolfendale, J. Phys. G 31, 255 (2005).

[57] V. S. Berezinsky, A. Gazizov, and S. Grigorieva, Phys. Rev. D 74, 043005 (2006).

[58] F. W. Stecker, M. A. Malkan, and S. T. Scully, Astrophys. J. 648, 043005 (2006).

[59] M. Ackermann et al. (Fermi-LAT Collaboration), Science 338, 1190 (2012).

[60] P. Biermann and P. Strittmatter, Astrophys. J. 322, 643 (1987).

[61] H. Yuksel and M. D. Kistler, Phys. Rev. D 75, 083004 (2007).

[62] H. Takami, K. Murase, S. Nagataki, and K. Sato, Astropart. Phys. 31, 201 (2009).

[63] G. Decerprit and D. Allard, Astro. Astrophys. 535, A66 (2011). 